\title{
Validating the HERDO02 rule to guide treatment duration for women with unprovoked venous thrombosis: multinational prospective cohort management study
}

Marc A Rodger, ${ }^{1,2,3}$ Gregoire Le Gal, 1,2,3,4 David R Anderson, ${ }^{5}$ Jeannot Schmidt 6 Gilles Pernod, Susan R Kahn, ${ }^{8}$ Marc Righini, ${ }^{9}$ Patrick Mismetti, ${ }^{10}$ Clive Kearon,, ${ }^{11}$ Guy Meyer, ${ }^{12}$ Antoine Elias, ${ }^{13}$ Tim Ramsay, ${ }^{2,3}$ Thomas L Ortel, ${ }^{14}$ Menno V Huisman, ${ }^{15}$ Michael J Kovacs, ${ }^{16}$ for the REVERSE II Study Investigators

\section{ABSTRACT}

OBJECTIVE

To prospectively validate the HERDOO2 rule

(Hyperpigmentation, Edema, or Redness in either leg; D-dimer level $\geq 250 \mu \mathrm{g} / \mathrm{L}$; Obesity with body mass index $\geq 30$; or Older age, $\geq 65$ years), which states that women with none or one of the criteria can safely discontinue anticoagulants after short term treatment. DESIGN

Prospective cohort management study.

SETTING

44 secondary or tertiary care centres in seven countries. PARTICIPANTS

Of 3155 consecutive eligible participants with a first unprovoked venous thromboembolism (VTE, proximal leg deep vein thrombosis or pulmonary embolism) who completed 5-12 months of short term anticoagulant treatment, 370 declined to participate, leaving 2785 enrolled participants. $2.3 \%$ were lost to follow-up.

INTERVENTIONS

Women with none or one of the HERDOO2 criteria were classified as at low risk of recurrent VTE and discontinued anticoagulants (intervention arm), whereas anticoagulant management for high risk women ( $\geq 2$ HERDO02 criteria) and men was left to the discretion of the clinicians and patients (observation arm).

MAIN OUTCOME MEASURE

Recurrent symptomatic VTE (independently and blindly adjudicated) over one year of follow-up.

\section{WHAT IS ALREADY KNOWN ON THIS TOPIC}

Current practice guidelines suggest that all patients with unprovoked venous thromboembolism (VTE) should continue anticoagulants long term unless they

have a high risk of bleeding

Most patients with unprovoked VTE will not, however, develop recurrent VTE

To date, no single predictor or combination of predictors (clinical decision rule) has been developed and validated to identify a group of patients with unprovoked VTE at sufficiently low risk of recurrent VTE to safely discontinue anticoagulants

\section{WHAT THIS STUDY ADDS}

In the primary analysis, 17 women identified as having a low risk of recurrent VTE using the HERDOO2 rule who discontinued anticoagulants developed recurrent VTE during 564 patient years of follow-up (3.0\% per patient year, $95 \%$ confidence interval $1.8 \%$ to $4.8 \%$ )

Using the HERDOO2 clinical decision rule allows clinicians to identify a large group of low risk group of women with unprovoked VTE who can safely discontinue anticoagulants

\section{RESULTS}

Of 1213 women, 631 (51.3\%) were classified as low risk and 591 discontinued oral anticoagulant treatment. In the primary analysis, 17 low risk women who discontinued anticoagulants developed recurrent VTE during 564 patient years of follow-up (3.0\% per patient year, $95 \%$ confidence interval $1.8 \%$ to $4.8 \%$ ). In 323

high risk women and men who discontinued anticoagulants, 25 had VTE during 309 patient years of follow-up (8.1\%, $5.2 \%$ to $11.9 \%$ ), whereas in 1802 high risk women and men who continued anticoagulants 28 had recurrent VTE during 1758 patient years of follow-up (1.6\%, $1.1 \%$ to $2.3 \%$ ).

\section{CONCLUSIONS}

Women with a first unprovoked VTE event and none or one of the HERDOO2 criteria have a low risk of recurrent VTE and can safely discontinue anticoagulants after completing short term treatment.

TRIAL REGISTRATION

clinicaltrials.gov NCT00967304.

\section{Introduction}

Venous thromboembolism (VTE), comprising deep vein thrombosis and pulmonary embolism, is a common, potentially fatal yet treatable condition. After short term oral anticoagulant treatment, the risk of recurrent VTE after discontinuing anticoagulants is variable, depending on the presence or absence of provoking factors at the time of the initial event. Some patient subgroups with major provoking factors (eg, post-surgical VTE) have a low risk of recurrent VTE ( $1 \%$ at one year $)^{1}$ and anticoagulants can be safely discontinued after short term treatment. ${ }^{2}$ However, more than $50 \%$ of patients with VTE do not have a major provoking factor. ${ }^{3}$ In these patients with unprovoked VTE, the risk of recurrent VTE is substantially higher; up to $10 \%$ in the first year after stopping short term treatment, 5\% in the subsequent year, ${ }^{4}$ and $30 \%$ at eight years. ${ }^{5}$ Recurrent VTE is fatal in $3.6 \%$ of cases. ${ }^{6}$

Oral anticoagulant treatment is effective at reducing the risk of recurrent VTE during treatment; oral anticoagulant treatment reduces the risk of recurrent VTE by 80-90\%. ${ }^{7}$ Longer term oral anticoagulant treatment likely only delays recurrent VTE compared with short term treatment. ${ }^{8-10}$ The highest bleeding risks are observed during initial oral treatment. Patients who do not bleed during this time, termed "anticoagulant experienced" patients, are at lower risk of major bleeding 
with ongoing treatment (1.3\% per year). ${ }^{7}$ Major bleeding is fatal in $11.3 \%$ of affected patients. ${ }^{6}$

Guidelines recommend long term anticoagulant treatment in patients with unprovoked VTE with nonhigh bleeding risks, but this is based on weak evidence (grade $2 \mathrm{~B})^{11}$ owing to closely balanced competing risks and burdens of recurrent VTE and major bleeding. Hence, the clinical question of who should continue taking anticoagulants indefinitely and who can safely discontinue them after short term treatment for unprovoked VTE remains a high research priority. ${ }^{910}$ To answer this important question, the long term risks and burdens of recurrent VTE must be balanced against the long term risks and burdens of oral anticoagulant treatment, especially major bleeding. The International Society on Thrombosis and Haemostasis suggests that it is safe to discontinue anticoagulants if the risk of recurrent VTE is less than 5\% at one year after discontinuing treatment. ${ }^{12}$ To date, no clinical decision rules ${ }^{13-15}$ designed to achieve these standards have been prospectively validated.

We previously derived a clinical decision rule to identify patients at low risk of recurrent VTE who could discontinue anticoagulants. ${ }^{15}$ The clinical decision rule, HERDOO2, identifies women with one or fewer of the four following criteria as being at low risk of recurrent VTE: Hyperpigmentation, Edema, or Redness in

HER: any Hyperpigmentation, Edema, or Redness in either leg (ie, mild, moderate, or severe). Assign 1 point for HER (ie, see visual guide below)

VIDAS D-dimer $\geq 250 \mu \mathrm{g} / \mathrm{L}$

Obesity (body mass index $\geq 30$ )

Older age ( $\geq 65$ years)

TOTAL $=$

Low risk: 0 or 1 point High risk: $\geq 2$ points

Visual guide:

Note: Signs may be less apparent in patients with brown or black skin

Hyperpigmentation
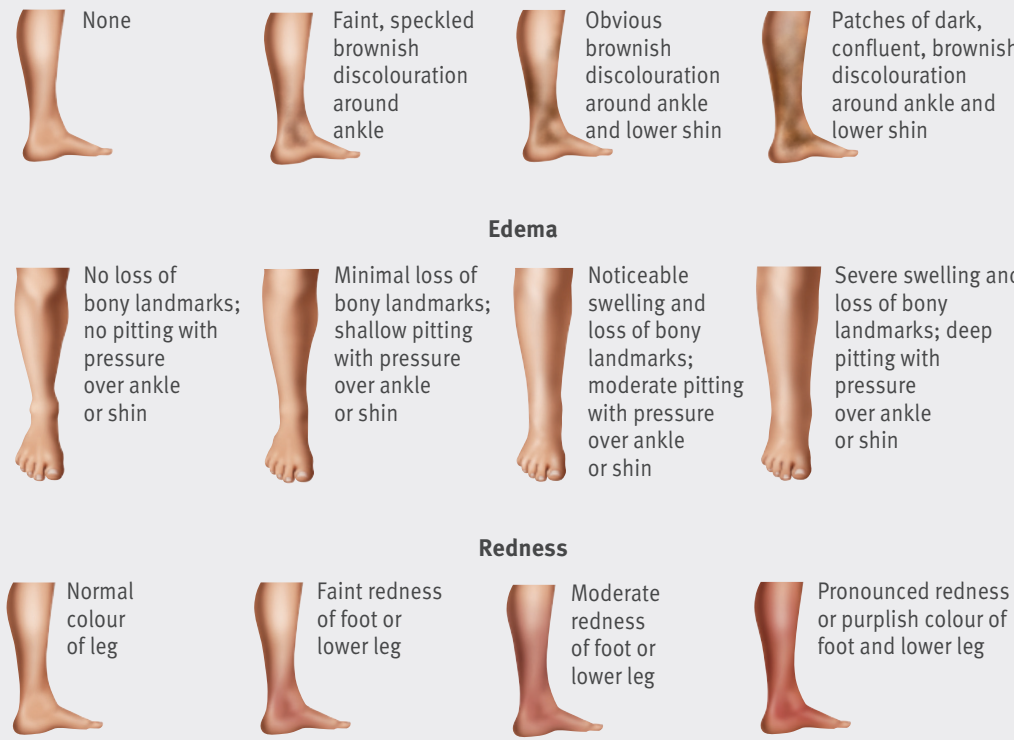

\section{Edema}
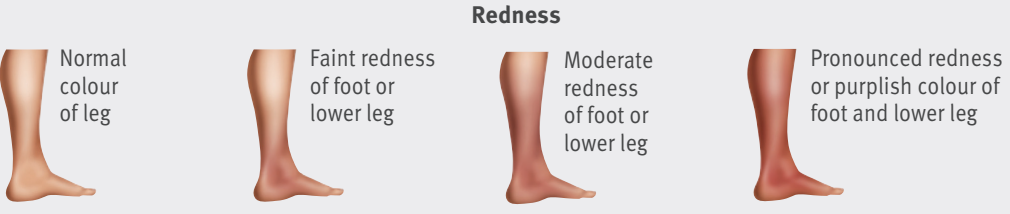

Fig 1 | HERD002 score to identify women with unprovoked venous thrombosis (VTE) at low risk of recurrent venous thrombosis. Visual guide adapted from Kahn et al ${ }^{16}$ either leg; D-dimer level $\geq 250 \mu \mathrm{g} / \mathrm{L}$ during treatment with anticoagulants; Obesity with body mass index $\geq 30$; and Older age, $\geq 65$ years (fig 1 ). ${ }^{15}$ No subgroup of men could be identified as low risk for recurrent VTE. Before adopting a clinical decision rule in clinical practice it should be prospectively validated in multiple clinical settings. ${ }^{17}$ As such, we validated the HERDOO2 clinical decision rule in a multinational prospective cohort management study.

\section{Methods}

\section{Study design}

We conducted a prospective multinational cohort management study of patients with a first unprovoked VTE event who were included after 5-12 months of anticoagulant treatment. Women were classified as low risk if they had none or one of the four HERDOO2 criteria (fig 1). Women who met two or more criteria and all men were classified as high risk. Per protocol, low risk patients were to discontinue anticoagulants (intervention arm), whereas anticoagulant management in high risk patients was left to the discretion of the clinicians and patients (observation arm). Participants were then followed for one year for recurrent VTE and major bleeding. All patients provided written informed consent before participation.

\section{Study participants and setting}

Consecutive unselected patients at 44 secondary and tertiary care centres in seven countries (see supplementary appendix) were asked to participate if they had a first episode of major, symptomatic, objectively proved unprovoked VTE 5-12 months before enrolment (referred to as the index VTE). Index VTE was defined as unprovoked, in line with other studies, ${ }^{18-20}$ in the absence of the following major VTE provoking factors: leg fracture or lower extremity plaster cast, immobilisation for more than three days, major surgery in the three months before the index VTE event, and no diagnosis of a malignancy in the past five years (with the exception of localised skin malignancy). Patients with malignancy arising after diagnosis of index VTE but before enrolment were not eligible for inclusion. Patients with previous episodes of provoked VTE were eligible. Index VTE associated with minor or weak risk factors such as travel, exogenous oestrogen, minor immobilisation, or minor surgery was considered unprovoked as it is unclear if the risk of recurrence in "weakly provoked" VTE is low enough to discontinue anticoagulants. ${ }^{12}$ Objective confirmation of index major VTE comprised either deep vein thrombosis demonstrated with a non-compressible segment in the popliteal vein or more proximal leg veins on compression venous ultrasonography and/or pulmonary embolism demonstrated on a high probability perfusion scan ${ }^{21}$ or a segmental or more proximal pulmonary artery filling defect on computed tomography (CT) pulmonary angiography. Index VTE had to have been managed for 5-12 months with an appropriate anticoagulant treatment, including initial treatment with either unfractionated heparin, low molecular weight heparin, rivaroxaban, or apixaban, 
followed by 5-12 months of oral anticoagulant treatment with vitamin $\mathrm{K}$ antagonists (target international normalised ratio 2-3), dabigatran, rivaroxaban, apixaban, or edoxaban.

Patients were excluded if they were unable or unwilling to provide informed consent; were aged less than 18 years; had already discontinued oral anticoagulant treatment; required ongoing anticoagulation (eg, mechanical heart valves, atrial fibrillation, inferior vena cava filter, or had known high risk thrombophilia before enrolment. High risk thrombophilia included deficiency of protein S, protein C, or antithrombin, persistently positive antiphospholipid antibodies, or two or more known other thrombophilic defects (eg, homozygous for factor $\mathrm{V}$ Leiden mutation or prothrombin gene mutation, or compound heterozygous for factor $\mathrm{V}$ Leiden and prothrombin gene mutation)); were geographically inaccessible for follow-up; had planned to use exogenous oestrogen (patch, ring, oral contraceptive, or hormone replacement therapy) if anticoagulant treatment was discontinued; or had pregnancy associated index VTE (antepartum or postpartum (within 12 weeks of delivery)).

\section{Standardised baseline patient assessment}

The baseline assessment took place 5-12 months after the index VTE event, while patients were still receiving oral anticoagulant treatment. We documented demographic data, causes, and diagnostic or therapeutic management of the index VTE event. In women, body mass index was calculated and both legs were examined for hyperpigmentation, oedema, or redness. D-Dimer samples were drawn during anticoagulant treatment at the baseline assessment (at any time if the patient was using vitamin $\mathrm{K}$ antagonists or at the time of peak anticoagulant effect for the other oral anticoagulants (dabigatran 4-8 hours, apixaban 2-6 hours, rivaroxaban 2-6 hours, and edoxaban 2-6 hours)). Within one hour of collection, plasma samples were double centrifuged at $2500 \times \mathrm{g}$ for 20 minutes and D-dimer testing was performed immediately. If testing was not available locally, samples were immediately frozen at $-70^{\circ} \mathrm{C}$ and transported on dry ice. D-dimer was measured using VIDAS D-dimer reagent on the VIDAS Instrument (bioMérieux, Marcy L'Etoile, France).

We then applied the HERDOO2 clinical decision rule. Per protocol, low risk women were to discontinue oral anticoagulant treatment, whereas continuation of anticoagulation in men and high risk women was left to the discretion of the clinicians and patients.

We also obtained baseline results for bilateral leg compression ultrasonography and either a ventilation-perfusion scan or perfusion only scan or CT pulmonary angiography. Baseline imaging was conducted to assist in the diagnosis of subsequent suspected recurrent VTE in patients who discontinued anticoagulants.

Follow-up and outcome assessment

Patients were contacted by telephone or seen in person at six months (two weeks either way). High risk patients were also telephoned or seen in person at one year (two weeks either way), whereas all low risk patients were seen in person at one year (two weeks either way). A structured interview for suspected VTE or bleeding was conducted at these visits. We also recorded the use of oral anticoagulant treatment. In addition to these visits, patients were instructed to contact study staff if they developed symptoms of recurrent VTE or bleeding at any time during follow-up. Participants were withdrawn and censored at the time they withdrew consent and did not permit the collection of further follow-up data, were lost to follow-up, or died.

\section{Outcomes}

The primary outcome was adjudicated symptomatic major VTE (new popliteal vein or more proximal deep vein thrombosis and/or new pulmonary embolism in at least a segmental or more proximal pulmonary artery). Secondary outcomes included major bleeding and mortality.

Two physicians independently adjudicated all available information about patients who died or were suspected of having an outcome (adjudicators were not from the same institution as the study participant). Adjudicators were blinded to the result of the clinical decision rule assessment and to the presence or absence of anticoagulant treatment. The criteria used for the diagnosis of recurrent deep vein thrombosis and pulmonary embolism were as previously published ${ }^{15}$ but in brief required documentation of new proximal deep vein thrombosis and/or segmental pulmonary embolism compared with baseline imaging (or index VTE imaging if baseline imaging was not available). ${ }^{22}$ Deaths were adjudicated and categorised as due to pulmonary embolism (certain, highly probable, possible, or unlikely), bleeding, or other. Major bleeds were defined as clinically overt bleeding associated with death or a decrease in haemoglobin concentration of more than $20 \mathrm{~g} / \mathrm{L}$ or transfusion of two or more units of whole blood or red blood cells or bleeding in a critical area or organ (intracranial, intraspinal, intraocular, retroperitoneal, intra-articular, pericardial, or intramuscular with compartment syndrome). ${ }^{23}$

\section{Statistical analysis}

We sought a sample size sufficient to ensure that the risk of recurrent major VTE in low risk patients was at most 3\% at one year (ie, upper bound of the confidence interval of the estimate of recurrent VTE at one year was $<3 \%$ ). To achieve this standard, at $80 \%$ power, with an anticipated rate of recurrent VTE of $1.8 \%$ at one year in low risk patients, we estimated we would require 828 low risk patients who discontinued anticoagulants (including a 9\% inflation for censored patients). After beginning enrolment in our study, the International Society on Thrombosis and Haemostasis suggested that a recurrent VTE risk threshold of less than 5\% at one year with an upper bound of the $95 \%$ confidence interval at $8 \%$ would be required to prove that patients with VTE could safely discontinue anticoagulants. ${ }^{12}$ In light of this more liberal threshold, the steering committee approved a revised sample size of 600 low risk patients 
who discontinued anticoagulants to more than adequately power the study to meet these recommended requirements.

In our a priori defined primary analysis, we determined the incidence of recurrent "major VTE" per patient year of follow-up in classifiable low risk patients who discontinued anticoagulants, and the exact binomial 95\% confidence interval around this rate. In a priori planned analyses of secondary outcomes, we explored the rate of major bleeding, death due to pulmonary embolism, and total mortality. In a priori planned subgroup analyses, we also explored the risk of recurrent major VTE and major bleeding in classifiable high risk patients who continued anticoagulants and in high risk patients who discontinued anticoagulants. Only complete case analysis was conducted (ie, only classifiable patients were analysed). All analyses were conducted with SAS V 9.3 software (SAS institute, Cary, NC).

\section{Patient involvement}

Patient partners were not involved in the design or conduct of this study that began in 2008. Patient partners in the CanVECTOR network (www.canvector.ca) will be involved in dissemination/knowledge translation activities.

\section{Results}

Between November 2008 and February 2015, we approached 3155 eligible participants, of whom 370 declined to participate, leaving 2785 enrolled participants in 44 centres in seven countries (fig 2). Of these, 2779 were classifiable with the HERDOO2 clinical decision rule and six were not (D-dimer result unavailable). Thirty two patients had no follow-up, leaving 2747 analysed patients with a mean age of 54.4 (SD 16.7 years), $44.3 \%$ were female, and $83.7 \%$ were white (table 1). In addition to the 32 patients with no follow-up, 31 patients had incomplete follow-up (63/2779 (2.3\% lost to follow-up)). Index first unprovoked VTEs were isolated deep vein thrombosis (40.5\%), isolated pulmonary embolism (38.9\%), and deep vein thrombosis and pulmonary embolism (20.6\%). Of 1213 women, 622 (51.3\%) were classified as low risk with the clinical decision rule and, of these, 591 discontinued anticoagulants and 31 continued treatment. Of 2125 high risk patients (1534 men and 591 high risk women), 1802 (84.8\%) continued anticoagulants and 323 (15.2\%) discontinued treatment (fig 2).

\section{Primary endpoint}

Seventy participants had recurrent major VTE, with one death from pulmonary embolism (case fatality rate $1.4 \%, 95 \%$ confidence interval $0.3 \%$ to $7.7 \%$ ). Of the 591 low risk women who discontinued anticoagulants, 17 developed recurrent major VTE during 564 patient years of follow-up (3.0\% recurrent VTE per patient year, 95\% confidence interval $1.8 \%$ to $4.8 \%$ ) (table 2). A low risk patient, in retrospect, noted to have persistent unexplained thrombocytosis from the time of index VTE, was subsequently confirmed to have JAK-2 positive essential thrombocytosis at the time of VTE recurrence without anticoagulant treatment.

One additional low risk woman developed cerebral vein thrombosis during one year follow-up without anticoagulant treatment (not in a priori defined primary outcome). In a post hoc sensitivity analysis, including

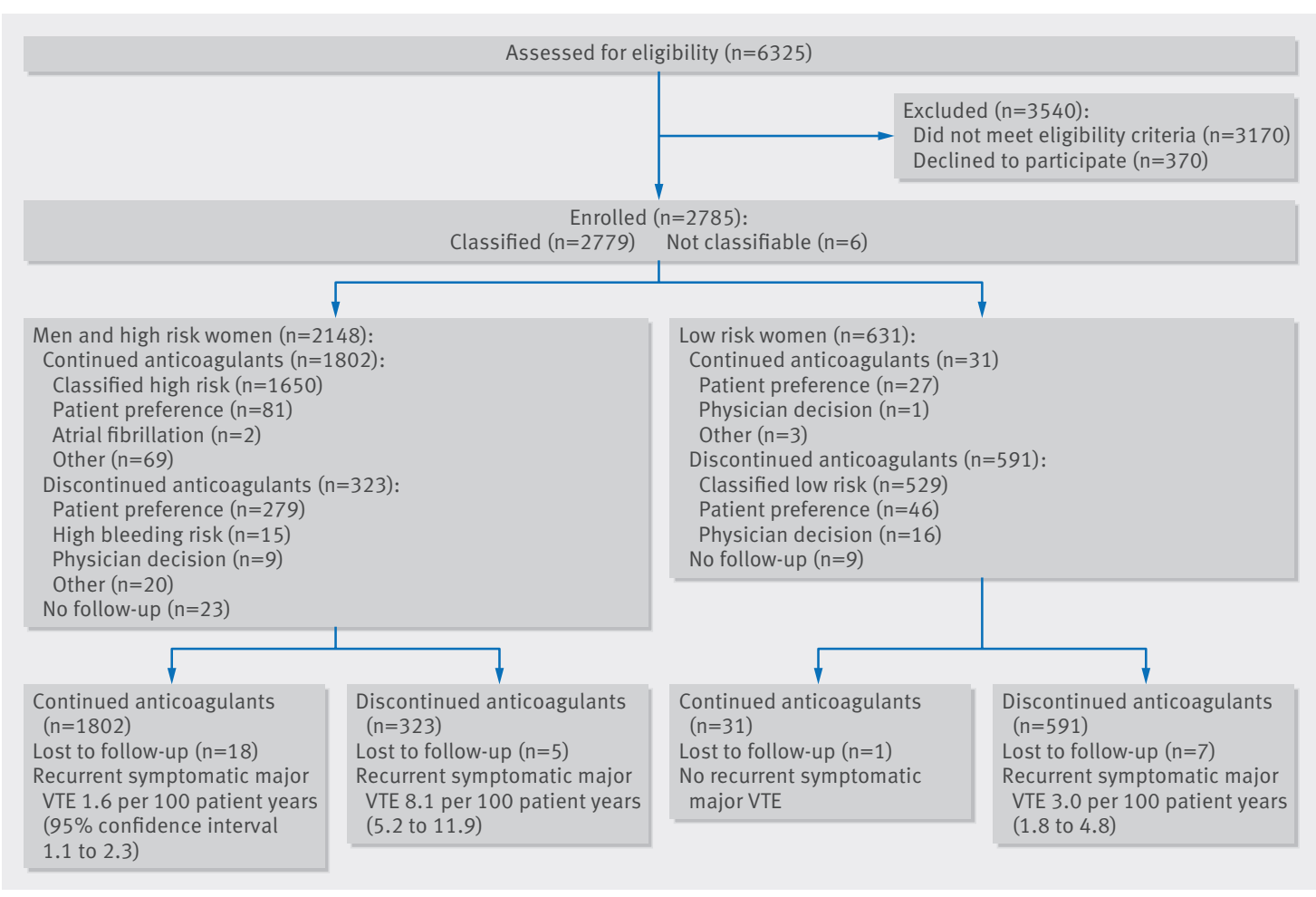

Fig 2 | Participant flow. VTE=venous thromboembolism 

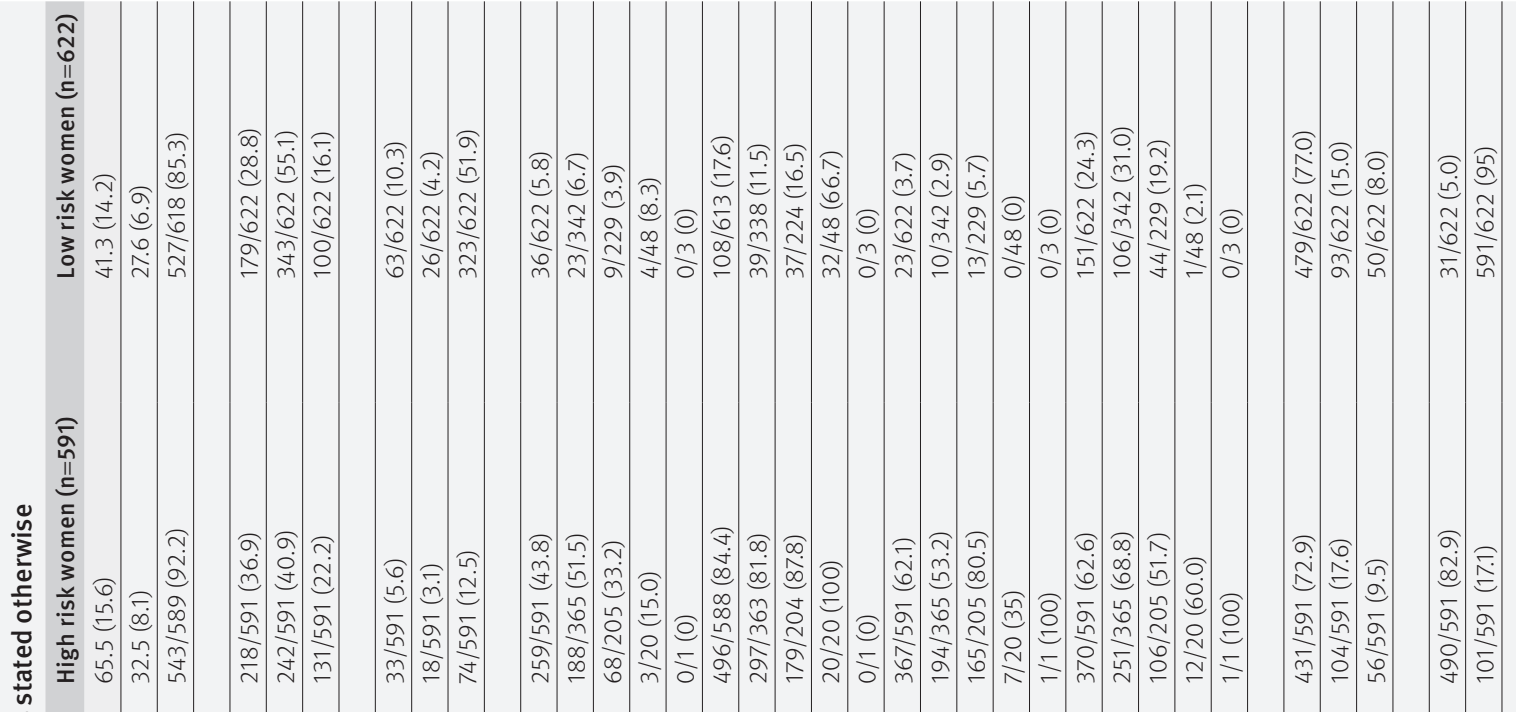

\section{$\frac{\breve{y}}{\Xi}$}

离

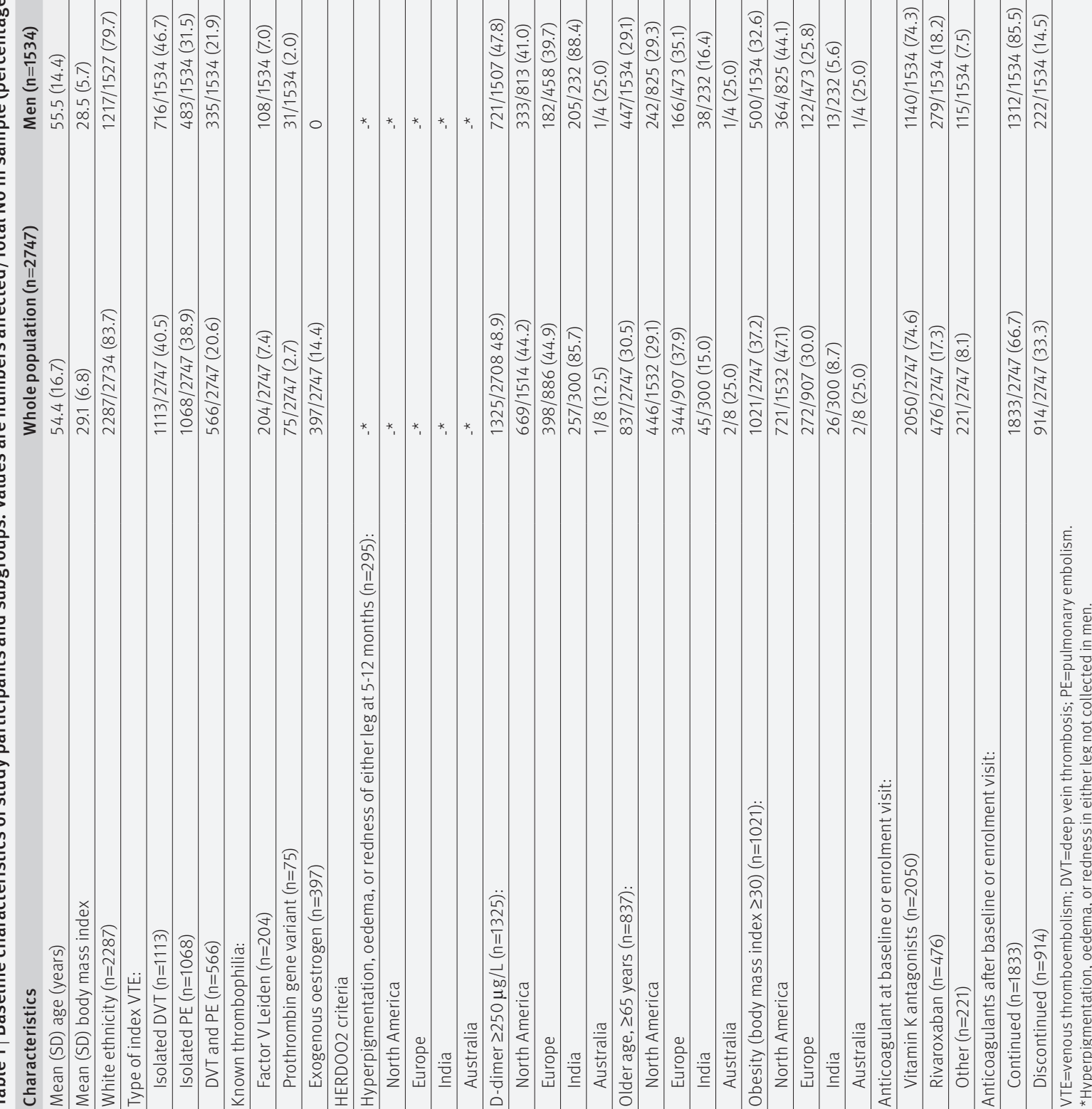




\begin{tabular}{|c|c|c|c|c|}
\hline Groups & $\begin{array}{l}\text { Low risk women* } \\
\text { who discontinued } \\
\text { oral anticoagulants } \\
(n=591)\end{array}$ & \multicolumn{2}{|c|}{ Men and high risk women* } & $\begin{array}{l}\text { High risk* women } \\
\text { who discontinued } \\
\text { oral anticoagulants } \\
(n=101)\end{array}$ \\
\hline \multicolumn{5}{|l|}{ Primary outcome: } \\
\hline \multicolumn{5}{|l|}{ Secondary outcomes: } \\
\hline Risk of major $\neq$ bleed & $0.2(0$ to 1.0$)$ & $0.6(0$ to 2.3$)$ & $1.2(0.8$ to 1.8$)$ & $2.1(0.3$ to 7.6$)$ \\
\hline Recurrent PE death & 0 & 0 & $0.1(0$ to 0.3$)$ & 0 \\
\hline Non-PE death & $0.2(0$ to 1.0$)$ & 0.1 .0 (0.2 to 2.8$)$ & $0.4(0.2$ to 0.8$)$ & $2.1(0.3$ to 7.6$)$ \\
\hline \multicolumn{5}{|c|}{$\begin{array}{l}\text { VTE=venous thromboembolism; } P E=\text { pulmonary embolism. } \\
\text { Not shown are results in low risk women who continued anticoagulants ( } n=31) \text {, patients with no follow-up }(n=32) \text {, or not classifiable owing to missing } \\
\text { D-dimer result }(n=6) \text {. } \\
\text { *HERD } 002 \text { criteria: hyperpigmentation, oedema, or redness in either leg at } 5-12 \text { months at baseline or enrolment visit, VIDAS D-dimer } \geq 250 \mu g / L \text { during } \\
\text { anticoagulant treatment, obesity with body mass index } \geq 30 \text {, or older age, } \geq 65 \text { years. Low risk=0 or } 1 \text { of the criteria present; high risk }=\geq 2 \text { or the criteria } \\
\text { present. } \\
\text { tProximal deep vein thrombosis and segmental or greater PE. } \\
\text { FAccording to definition by International Society on Thrombosis and Haemostasis. }{ }^{23}\end{array}$} \\
\hline
\end{tabular}

cerebral vein thrombosis in a modified primary outcome, the risk of recurrent VTE in low risk women would have been $3.2 \%$ (95\% confidence interval $2.0 \%$ to $5.0 \%$ ). In a second post hoc "worst case scenario" sensitivity analysis, assuming all low risk patients who discontinued anticoagulants who were lost to follow-up $(n=16)$ developed recurrent VTE and including cerebral vein thrombosis in a modified primary outcome, the risk of recurrent VTE in low risk women would have been $5.7 \%$ (4.1\% to $7.8 \%)$.

In 323 high risk patients who discontinued anticoagulants, 25 recurrent VTE events occurred during 309 patient years of follow-up (8.1\% recurrent VTE per patient year, 95\% confidence interval 5.2\% to $11.9 \%$ ). The risk of recurrent major VTE in high risk women who discontinued anticoagulants (7.4\% 3.0\% to $15.3 \%$ ) was comparable with men who discontinued anticoagulants $(8.4 \%, 5.0 \%$ to $13.2 \%)$. Twenty eight high risk patients who continued anticoagulants had recurrent VTE during 1758 patient years of follow-up (1.6\% recurrent VTE per patient year, 95\% confidence interval 1.1\% to $2.3 \%$ ) (see table 2).

\section{Bleeding events}

Twenty two major bleeds occurred during 1788 person years of follow-up in patients who continued anticoagulants (1.2\% major bleeds per patient year, 95\% confidence interval $0.8 \%$ to $1.9 \%$ ) compared with three major bleeds during 874 years of follow-up in patients who discontinued anticoagulants (0.3\% major bleeds per patient year, $0.1 \%$ to $1.0 \%$ ). Two major bleeds resulted in death (major bleed case fatality rate was $8.0 \%, 95 \%$ confidence interval $2.2 \%$ to $25.0 \%$ ).

\section{Subgroup analyses}

Of 429 low risk women of premenopausal age $(<50$ years) who discontinued anticoagulants, the risk of recurrent VTE was low regardless of whether their index VTE was associated with oestrogen $(n=291)(1.4 \%$ per patient year, $95 \%$ confidence interval $0.4 \%$ to $3.7 \%$ ) or not associated with oestrogen $(\mathrm{n}=138)(3.1 \%$ per patient year, $0.8 \%$ to $7.9 \%$ ) (table 3 ). In contrast, the risk of

recurrent VTE was 5.7\% per patient year (95\% confidence interval $2.6 \%$ to $10.9 \%$ ) in low risk women of postmenopausal age ( $\geq 50$ years) who discontinued anticoagulants $(\mathrm{n}=162)$.

In additional subgroup analyses, there were no differences in the rate of recurrent VTE over one year follow-up in low risk women who discontinued anticoagulants according to 1) country or region of enrolment, 2) type of index VTE (isolated deep vein thrombosis, isolated

Table 3 | Subgroup analysis with primary outcome in risk group by continued or discontinued anticoagulants in events $(\%)$ per 100 patient years $(95 \%$ confidence intervals)

\begin{tabular}{|c|c|}
\hline Groups & $\begin{array}{l}\text { Low risk women* } \\
\text { who discontinued } \\
\text { oral anticoagulants } \\
(n=591)\end{array}$ \\
\hline Age <50 $(n=429)$ : & $2.0(0.8$ to 3.9$)$ \\
\hline Oestrogen $(n=291)$ & $1.4(0.4$ to 3.7$)$ \\
\hline No oestrogen $(n=138)$ & $3.1(0.8$ to 7.9$)$ \\
\hline Age $\geq 50(n=162)$ & 5.7 (2.6 to 10.9$)$ \\
\hline Oestrogen $(n=24)$ & 0 \\
\hline No oestrogen $(n=138)$ & 6.8 (3.1 to 12.8$)$ \\
\hline \multicolumn{2}{|l|}{ Type of index VTE: } \\
\hline Isolated DVT (n=177) & $3.0(1.0$ to 7.0$)$ \\
\hline Isolated PE (n=323) & $3.9(2.0$ to 6.8$)$ \\
\hline DVT and PE $(n=91)$ & 0 \\
\hline \multicolumn{2}{|l|}{ Country or region: } \\
\hline North America $(n=334)$ & 3.7 (1.9 to 6.6$)$ \\
\hline Europe $(n=207)$ & $2.5(0.1$ to 5.9$)$ \\
\hline India $(n=47)$ & 0 \\
\hline Australia $(n=3)$ & 0 \\
\hline \multicolumn{2}{|l|}{ Anticoagulant at baseline or enrolment visit: } \\
\hline Vitamin K antagonist $(\mathrm{n}=459)$ & $2.9(1.6$ to 5.0$)$ \\
\hline All non-vitamin K antagonists $(\mathrm{n}=132)$ & $3.2(0.9$ to 8.3$)$ \\
\hline \multicolumn{2}{|l|}{ Known “weak" thrombophilia: } \\
\hline Factor V Leiden $(n=59)$ & $3.5(0.4$ to 12.6$)$ \\
\hline Prothrombin gene variant $(n=26)$ & $3.9(0.1$ to 21.6$)$ \\
\hline \multicolumn{2}{|c|}{$\begin{array}{l}\text { VTE=venous thromboembolism; CVT=deep vein thrombosis; } \\
\text { PE = pulmonary embolism. } \\
\text { *HERDO02 criteria: hyperpigmentation, oedema, or redness in either leg } \\
\text { at } 5-12 \text { months at baseline or enrolment visit, VIDAS D-dimer } \geq 250 \mu \mathrm{g} / \mathrm{L} \\
\text { during anticoagulant treatment, obesity with body mass index } \geq 30 \text {, or } \\
\text { older age, } \geq 65 \text { years. Low risk }=0 \text { or } 1 \text { of the criteria present. }\end{array}$} \\
\hline
\end{tabular}


pulmonary embolism, deep vein thrombosis and pulmonary embolism), or 3) type of anticoagulant treatment at the time of baseline visit (table 3).

\section{Discussion}

We prospectively validated a clinical decision rule to identify low risk patients who can safely discontinue anticoagulants after completing 5-12 months of treatment for a first unprovoked VTE. Women with none or one of the HERDOO2 criteria had a low risk of recurrent VTE and can safely discontinue anticoagulants after completing short term treatment. This is an important finding because using our rule over half of women with unprovoked VTE can safely discontinue anticoagulants and be spared the burdens, costs, and risks of indefinite anticoagulation.

The question of duration of anticoagulation for the prevention of recurrent VTE after a first unprovoked event is controversial. ${ }^{24}$ Updated consensus guidelines suggest anticoagulants should be continued indefinitely in patients with unprovoked VTE with a non-high bleeding risk. ${ }^{1125}$ We have shown that half of women with unprovoked VTE, regardless of bleeding risk, can safely discontinue anticoagulants. The International Society on Thrombosis and Haemostasis suggests that it is appropriate to stop anticoagulants in subgroups with a point estimate of recurrent VTE at one year below $5 \%$ if the upper bound of the $95 \%$ confidence interval is less than $8 \% .{ }^{12}$ In our study, we have shown that the one year recurrent VTE point estimate and the upper bound of the $95 \%$ confidence interval in low risk women with one or none of the HERDOO2 criteria are both less than 5\%.

Several individual predictors that seem to stratify the risk of recurrent VTE in patients with unprovoked index VTE have been identified, including sex, plasma D-dimer levels measured 1-2 months after discontinuation of oral anticoagulant treatment, ${ }^{26}$ and residual venous obstruction using venous ultrasonography. ${ }^{2728}$ None of these individual predictors appears to adequately identify a subgroup of patients with unprovoked VTE with a sufficiently low risk of recurrence to safely discontinue anticoagulants. Other multivariable clinical decision rules have been developed but have not been prospectively validated, including the Vienna prediction model $^{13}$ and the DASH score. ${ }^{14}$

Whether men and high risk women require indefinite anticoagulation remains controversial and was not the primary focus of our study. However, future research to identify additional low risk subgroups who may be able to also discontinue anticoagulants, will be important given that a large proportion of "high risk" patients will not develop recurrent VTE during non-use of oral anticoagulants. For example, in our long term follow-up study of the derivation group for our clinical decision rule, the risk of VTE in men and high risk women during eight years' follow-up was less than $40 \%$ (ie, $>60 \%$ did not recur in the long term). 5

Strengths and limitations of this study

Strengths of this study include the homogenous patient population (we only included symptomatic major VTE and excluded distal deep vein thrombosis and subsegmental pulmonary embolism) treated uniformly with short duration anticoagulant treatment (5-12 months). We applied our clinical decision rule prospectively in patients in whom the clinical decision is uncertain (patients with weakly provoked and unprovoked VTE) and at the time point that clinicians generally use to assist patients in making this decision about long term treatment. The large sample size of the study permitted precise estimates of recurrence in low risk patients. Most low risk patients stopped anticoagulants (95\%) as planned, suggesting the rule was acceptable to clinicians and patients. Our rule, consisting of a limited number of simple elements previously shown to have high interobserver reliability, ${ }^{29}$ was applied by a wide variety of clinicians in a wide variety of settings in multiple countries, and performed well. We had minimal loss to follow-up (2.3\%). All of our outcomes were symptomatic, objectively documented, and independently adjudicated compared with baseline imaging without knowledge of clinical decision rule classification or oral anticoagulant treatment. We included patients whose VTE was treated with vitamin $\mathrm{K}$ antagonists and direct oral anticoagulants, providing reassurance that our rule can be used with all contemporary treatment management strategies. We are optimistic that low risk women (none or one of the HERDOO2 criteria) will continue to be low risk in longer term follow-up as we showed in our derivation study. ${ }^{5}$ It is likely that low risk women will have a low risk of recurrent VTE over longer term follow-up because patients with unprovoked VTE have a lower risk of recurrent VTE in the years that follow the first year without anticoagulants. ${ }^{5}$ None the less, longer term follow-up of low risk women (none or one of the HERDOO2 criteria) should be the object of future research. Finally, study characteristics, participant characteristics, and outcomes were comparable between our derivation study population and validation study population (table 4).

Our work also has limitations. Firstly, in our derivation study we chose to explore all known potential predictors of recurrent VTE and we used complete case analysis rather than imputation of missing data in model building. We also chose to dichotomise our potential predictors at optimal cut points, minimise the final number of predictors, and use a simple scoring system to make the rule easy to use and more likely to be adopted in practice. However, by modeling with a dataset that contained a low event per predictor variable ratio, not imputing missing data, dichotomising variables at optimal cut points, and not using the exact or representative predictor variable weightings, we potentially derived a less powerful model and one that risked being over-fit. However, by validating our simple model in the current study we have shown that the HERDOO2 rule was not overfit. Secondly, patients with known "high risk" thrombophilia were excluded hence our findings may not apply to these patients. Thirdly, we used a single 


\begin{tabular}{|c|c|c|}
\hline Variables & Derivation study $(\mathrm{n}=646)$ & Validation study $(\mathrm{n}=2747)$ \\
\hline \multicolumn{3}{|l|}{ Study characteristics } \\
\hline Study design & Prospective cohort & Prospective cohort \\
\hline Timeline & $2001-06$ & $2008-15$ \\
\hline Setting & 12 tertiary centres in four countries & 44 secondary and tertiary centres in seven countries \\
\hline Inclusion criteria & $\begin{array}{l}\text { First objectively proved; unprovoked* major } \\
\text { VTE† treated with anticoagulants for 5-7 months }\end{array}$ & $\begin{array}{l}\text { First objectively proved; unprovoked* major VTEt treated with } \\
\text { anticoagulants for 5-12 months }\end{array}$ \\
\hline Exclusion criteria & $\begin{array}{l}\text { Recurrent VTE during treatment; discontinued } \\
\text { anticoagulants; require ongoing anticoagulants; } \\
\text { known "high risk" thrombophilia }\end{array}$ & $\begin{array}{l}\text { Recurrent VTE during treatment; discontinued anticoagulants; } \\
\text { require ongoing anticoagulants; known "high risk" thrombophilia; } \\
\text { plan on using exogenous oestrogen after stopping } \\
\text { anticoagulants; pregnancy associated index VTE event }\end{array}$ \\
\hline Primary outcome & $\begin{array}{l}\text { Blind independent adjudicated recurrent major } \\
\text { VTE† compared with baseline imaging }\end{array}$ & $\begin{array}{l}\text { Blind independent adjudicated recurrent major VTE† compared } \\
\text { with baseline imaging }\end{array}$ \\
\hline Anticoagulation treatment after enrolment & Stopped in all participants & $\begin{array}{l}\text { Low risk women (0 or } 1 \text { HERDOO2 criteria): anticoagulants } \\
\text { stopped per protocol } \\
\text { High risk women and men ( } \geq 2 \text { HERDOO2 criteria): anticoagulant } \\
\text { decision left to clinicians and patients (ie, observation only) } \\
\text { ( } 84.8 \% \text { continued anticoagulants) }\end{array}$ \\
\hline Mean follow-up (months) & 18.0 & 11.6 \\
\hline \multicolumn{3}{|l|}{ Participant characteristics } \\
\hline Men & $332 / 646(51.4)$ & 1534/2747 (55.8) \\
\hline Mean age (years) & 52.5 & 54.4 \\
\hline White & $597 / 646(92.4)$ & $2287 / 2734(83.7)$ \\
\hline Women with oestrogen associated index VTE & $73 / 322(22.6)$ & $397 / 1213(32.7)$ \\
\hline \multicolumn{3}{|l|}{ Index VTE type: } \\
\hline Isolated DVT & $339 / 646(52.5)$ & $1113 / 2747(40.5)$ \\
\hline Isolated PE & $194 / 646(30.0)$ & 1068/2747 (38.9) \\
\hline DVT and PE & $113 / 646(17.5)$ & $566 / 2747(20.6)$ \\
\hline
\end{tabular}

HERDOO2 predictors in female participants at baseline examination:

\begin{tabular}{|c|c|c|}
\hline Hyperpigmentation, oedema, or redness in either leg & $96 / 260(36.9)$ & $295 / 1213(24.3)$ \\
\hline D-dimer $\geq 250 \mu \mathrm{g} / \mathrm{L}$ during anticoagulant use & $121 / 304(39.8)$ & $604 / 1201(50.3)$ \\
\hline Older age, $\geq 65$ years & $87 / 314(27.7)$ & $390 / 1213(32.2)$ \\
\hline Obesity with $\mathrm{BMI} \geq 30$ & $114 / 313(36.4)$ & $521 / 1213(43.0)$ \\
\hline 0 or 1 & $164 / 314(52.2)$ & $622 / 1213(51.3)$ \\
\hline$\geq 2$ & $150 / 314(47.8)$ & $591 / 1213(48.7)$ \\
\hline \multicolumn{3}{|l|}{$\begin{array}{l}\text { Primary outcome event rates (recurrent major VTE (\%) } \\
\text { per } 100 \text { patient years }(95 \% \mathrm{CI} \text { ( No affected)): }\end{array}$} \\
\hline \multicolumn{3}{|l|}{ Men: } \\
\hline Discontinue anticoagulants & $13.7(10.8$ to 17.0$)(n=332)$ & $8.4(5.0$ to 13.2$)(n=222)$ \\
\hline Continue anticoagulants & $-(n=0)$ & $1.2(0.7$ to 2.0$)(n=1312)$ \\
\hline \multicolumn{3}{|l|}{ High risk women ( $\geq 2$ HERDOO2 criteria): } \\
\hline Discontinue anticoagulants & $14.1(10.9$ to 17.3$)(n=150)$ & $7.4(3.0$ to 15.3$)(n=101)$ \\
\hline
\end{tabular}

VTE=venous thromboembolism; DVT=deep vein thrombosis; $\mathrm{PE}=$ pulmonary embolism; $\mathrm{BMI}=$ body mass index.

*Absence of leg fracture or leg cast or major surgery in past three months, and no malignancy in past five years. tProximal DVT and/or segmental or greater PE.

D-dimer reagent and instrument (VIDAS D-dimer, bioMérieux, Marcy L'Etoile, France) at half of the usual cut point for D-Dimer testing that is used in diagnostic management. It is uncertain if other D-dimer assays can be used in our clinical decision rule. Fourthly, some subgroups require further elaboration. In women of premenopausal age ( $<50$ years) with none or one of the HERDOO2 criteria who discontinued anticoagulants, the risk of recurrent VTE was low $(<5 \%$ at one year) regardless of whether or not these women were using exogenous oestrogen at the time of their index VTE event (table 3 ). These results are consistent with previous studies, which suggest that women with oestrogen associated VTE have a comparable risk of recurrent VTE to age matched women with non-oestrogen associated VTE, suggesting that oestrogen is not an important independent predictor of recurrent VTE in otherwise unprovoked VTE. ${ }^{30} 31$ Furthermore, we have previously shown that women with oestrogen associated VTE with a low HERDOO2 score had an eightfold lower risk of recurrent VTE than women with oestrogen associated VTE 
and a high HERDOO2 score, highlighting the value of using HERDOO2 for risk stratification in women with oestrogen associated VTE. ${ }^{30}$ In contrast, in the subgroup of women of postmenopausal age ( $\geq 50$ years) with none or one of the HERDOO2 criteria who discontinued anticoagulants, the risk of recurrent VTE was higher than expected (point estimate 5.7\%). As such, further validation of HERDOO2 is required in the subset of postmenopausal women. Finally, we had a limited number of non-white participants and as such our clinical decision rule should be further validated in other populations.

\section{Conclusion}

Women with none or one of the four HERDOO2 criteria: hyperpigmentation, oedema, or redness in either leg, D-dimer level $\geq 250 \mu \mathrm{g} / \mathrm{L}$ while using anticoagulants, obesity with body mass index $\geq 30$, or older age, $\geq 65$ years) have a low risk of recurrent VTE and can safely discontinue anticoagulants after completing short term anticoagulants after a first unprovoked VTE event. Further research is required to firmly establish the safety of using HERDOO2 to identify low risk non-white women and women aged more than 50 years.

\section{AUTHOR AFFILIATIONS}

'Thrombosis Program, Division of Hematology, Ottawa Blood Disease Center, Department of Medicine, Faculty of Medicine University of Ottawa, Ottawa, ON, K1H 8L6, Canada

${ }^{2}$ Clinical Epidemiology Program, Ottawa Hospital Research Institute, Ottawa, ON, Canada

${ }^{3}$ School of Epidemiology, Public Health and Preventative Medicine, University of Ottawa, Ottawa, ON, Canada

4EA 3878, INSERM CIC 1412, Université de Brest, Brest, France ${ }^{5}$ Faculty of Medicine, Dalhousie University, Halifax, NS, Canada ${ }^{6}$ Centre Hospitalier Universitaire de Clermont-Ferrand, Clermont, France ${ }^{7}$ Centre Hospitalier Universitaire de Grenoble, Grenoble, France ${ }^{8}$ Jewish General Hospital, McGill University, Montreal, QC, Canada 9Division of Angiology and Hemostasis, Geneva University Hospital, Geneva, Switzerland

${ }^{10} \mathrm{Centre}$ Hospitalier Universitaire de Saint Etienne Bellevue, Saint Etienne, France

${ }^{11}$ Department of Medicine and Thrombosis and Atherosclerosis Research Institute, McMaster University, Hamilton, ON, Canada

${ }^{12}$ Respiratory Department, Hopital Europeen Georges-Pompidou, APHP, Université Paris Descartes Sorbonne, Paris Cité: INSERM UMRS 970, Paris, and INNOVTE, Saint-Etienne, France

${ }^{13}$ Centre Hospitalier Intercommunal de Toulon, Toulon, France ${ }^{14}$ Department of Medicine, Duke University Medical Center, Durham, NC, USA

${ }^{15}$ Department of Thrombosis and Hemostasis, Leiden University Medical Center, Leiden, Netherlands

${ }^{16}$ London Health Sciences Centre, Western University, London, ON, Canada

We thank the staff and patients from the thrombosis clinics who participated in the study; the central study team, including Tammy Beaudoin, Marisol Betancourt, Faizan Khan, Anne Marie Clement, Megan Inskip, and Isabelle Pichon; and Wim Houdijk, Alicia Rico-Lazarowski, and Franck Berthier from bioMerieux for support throughout study development and implementation. In France, participating centres are members of the INNOVTE Network; in Canada, participating centres are members of the CanVECTOR Network; and in Australia, participating members are members of the Australasian Society of Thrombosis and Hemostasis Clinical Trials Group. These national networks are members of INVENT-VTE, the International Network of Venous Thromboembolism Clinical Research Networks (www.invent-VTE.com). The results reported in this paper were presented in part at the European Society of Cardiology meeting in August 2016 in Rome, Italy.

Contributors: MAR (lead investigator worldwide) conceived the study; obtained peer reviewed and non-peer reviewed funding; chaired the steering committee; wrote the first draft of the protocol; recruited patients; wrote the first draft of the manuscript; developed the analysis plan; and interpreted the study results. GLeG (lead investigator in France) conceived the study; obtained peer reviewed and non-peer reviewed funding; was a member of the steering committee; wrote the first draft of the protocol and first draft of the manuscript; developed the analysis plan; and interpreted the study results. MJK conceived the study; obtained non-peer reviewed funding; was a member of the steering committee; wrote the first draft of the protocol and first draft of the manuscript; developed the analysis plan; and interpreted the study results. DRA and SRK contributed to the study design and recruited patients. IS, GP, PM, CK, and GM were responsible for local study implementation and recruited patients. MR and TLO contributed to the study design; recruited patients; and was a member of the steering committee. TR contributed to the study design, particularly the data analysis plan (lead statistician) and supervised the statistical analysis. AE recruited patients. MVH contributed to the study design and was a member of the steering committee. All authors critically revised and approved the final manuscript.

REVERSE II Study Investigators were responsible for local implementation of the study; recruited patients; and critically revised and approved the final manuscript.

REVERSE II study adjudicators reviewed suspected study events and critically revised and approved the final manuscript.

REVERSE II study data safety monitoring committee reviewed study safety reports and critically revised and approved the final manuscript.

Funding: MAR holds a career investigator award from the Heart and Stroke Foundation of Canada (Cl 7441) and the University of Ottawa Faculty of Medicine clinical research chair in thrombosis and thrombophilia. CK holds a career investigator award from the Heart and Stroke Foundation of Canada (Cl 7438) and the Jack Hirsh professorship in thromboembolism. SRK is a tier 1 Canada research chairholder. GLeG holds a clinician scientist award from the Heart and Stroke Foundation of Ontario and the University of Ottawa Faculty of Medicine clinical research chain in diagnosis of venous

thromboembolism. This investigator initiated study was sponsored by the Ottawa Hospital Research Institute and funded by a grant from the French Ministry of Health (PHRC 2009-08-05) and bioMérieux. MAR, TR, and Ranjeeta Mallick had full access to all study data and take responsibility for the integrity of the data and the accuracy of the data analysis. MAR, TR, and Ranjeeta Mallick from the Ottawa Hospital Research Institute conducted the analyses, independent of the funders. The funders did not have access to the data and did not influence the decision to publish.

Competing interests: All authors have completed the ICMIE uniform disclosure form at www.icmje.org/coi_disclosure.pdf and declare: the study was partially funded by bioMérieux the manufacturer of the Vidas D-Dimer (used in the current study); no financial relationships with any organisations that might have an interest in the submitted work in the previous three years; no other relationships or activities that could appear to have influenced the submitted work. All authors fulfilled the conditions required for authorship and the final manuscript has been seen and approved by all authors (see authorship appendix for detailed contributions).

Ethical approval: Institutional research ethics board approval was obtained in the lead centre (Ottawa Health Sciences Network Research Ethics Board ID No 2008039-01H) and at all participating centres.

Data sharing: De-anonymised patient level data and the full dataset with low risk of identification are available on reasonable request after approval by the trial steering committee, Ottawa Health Research Institute, and Ottawa Health Sciences Network research ethics board.

Transparency: The lead author (MAR) affirms that the manuscript is an honest, accurate, and transparent account of the study being reported; that no important aspects of the study have been omitted; and that any discrepancies from the study as planned have been explained. No medical writer was engaged to write any part of this manuscript.

This is an Open Access article distributed in accordance with the Creative Commons Attribution Non Commercial (CC BY-NC 4.0) license, 
which permits others to distribute, remix, adapt, build upon this work non-commercially, and license their derivative works on different terms, provided the original work is properly cited and the use is non-commercial. See: http://creativecommons.org/licenses/ by-nc/4.0/.

1 Baglin T, Luddington R, Brown K, Baglin C. Incidence of recurrent venous thromboem bolism in relation to clinical and thrombophilic risk factors: prospective cohort study. Lancet 2003;362:523-6. doi:10.1016/S0140-6736(03)14111-6.

2 Kearon C, AkI EA, Comerota Al, et al. American College of Chest Physicians. Antithrombotic therapy for VTE disease: Antithrombotic Therapy and Prevention of Thrombosis, 9th ed. Chest 2012;141(Suppl):e419S-94S

3 White RH. The epidemiology of venous thromboembolism. Circulation 2003;107(Suppl 1):14-8. doi:10.1161/01.CIR.0000078468. 11849.66.

4 Agnelli G, Prandoni P, Santamaria MG, et al. Warfarin Optimal Duration Italian Trial Investigators. Three months versus one year of oral anticoagulant therapy for idiopathic deep venous thrombosis. N Engl J Med 2001;345:165-9. doi:10.1056/NEJM200107193 450302

5 Rodger MA, Scarvelis D, Kahn SR, et al. Long-term risk of venous thrombosis after stopping anticoagulants for a first unprovoked event: A multi-national cohort. Thromb Res 2016;143:152-8. doi:10.1016/j. thromres.2016.03.028

6 Carrier M, Le Gal G, Wells PS, Rodger MA. Systematic review: case-fatality rates of recurrent venous thromboembolism and major bleeding events among patients treated for venous thromboembolism. Ann Intern Med 2010;152:578-89. doi:10.7326/0003-4819-152-9-201005040-00008.

7 Castellucci LA, Cameron C, Le Gal G, et al. Efficacy and safety outcomes of oral anticoagulants and antiplatelet drugs in the secondary prevention of venous thromboembolism: systematic review and network meta-analysis. BMJ 2013;347:f5133. doi:10.1136/ bmj. 55133.

8 Couturaud F, Sanchez O, Pernod G, et al. PADIS-PE Investigators. Six months vs extended oral anticoagulation after a first episode of pulmonary embolism: The PADIS-PE randomized clinical trial. JAMA 2015;314:31-40. doi:10.1001/jama.2015.7046.

9 Agnelli G, Prandoni P, Becattini C, et al. Warfarin Optimal Duration Italian Trial Investigators. Extended oral anticoagulant therapy after first episode of pulmonary embolism. Ann Intern Med 2003;139:1925. doi:10.7326/0003-4819-139-1-200307010-00008.

10 Pinede L, Ninet J, Duhaut P, et al. Investigators of the "Durée Optimale du Traitement AntiVitamines K” (DOTAVK) Study. Comparison of 3 and 6 months of oral anticoagulant therapy after a first episode of proximal deep vein thrombosis or pulmonary embolism and comparison of 6 and 12 weeks of therapy after isolated calf deep vein thrombosis. Circulation 2001;103:2453-60. doi:10.1161/01. CIR.103.20.2453.

11 Kearon C, Akl EA, Ornelas J, et al. Antithrombotic Therapy for VTE Disease: CHEST Guideline and Expert Panel Report. Chest 2016;149:315-52. doi:10.1016/j.chest.2015.11.026.

12 Kearon C, Iorio A, Palareti G. Subcommittee on Control of Anticoagulation of the SSC of the ISTH. Risk of recurrent venous thromboembolism after stopping treatment in cohort studies: recommendation for acceptable rates and standardized reporting. J Thromb Haemost 2010;8:2313-5. doi:10.1111/j.1538-7836.2010.03991.x

13 Eichinger S, Heinze G, Jandeck LM, Kyrle PA. Risk assessment of recurrence in patients with unprovoked deep vein thrombosis or pulmonary embolism: the Vienna prediction model. Circulation 2010:121:1630-6. doi:10.1161/CIRCULATIONAHA.109.925214.

14 Tosetto A, Iorio A, Marcucci M, et al. Predicting disease recurrence in patients with previous unprovoked venous thromboembolism: a proposed prediction score (DASH). J Thromb Haemost 2012;10:101925. doi:10.1111/j.1538-7836.2012.04735.x.

15 Rodger MA, Kahn SR, Wells PS, et al. Identifying unprovoked thromboembolism patients at low risk for recurrence who can discontinue anticoagulant therapy. CMAJ 2008;179:417-26. doi:10.1503/ cmaj.080493.
16 Kahn SR, Partsch H, Vedantham S, Prandoni P, Kearon C. Subcommittee on Control of Anticoagulation of the Scientific and Standardization Committee of the International Society on Thrombosis and Haemostasis. Definition of post-thrombotic syndrome of the leg for use in clinical investigations: a recommendation for standardization. J Thromb Haemost 2009;7:879-83.doi:10.1111/j.1538-7836.2009.03294.x.

17 McGinn TG, Guyatt GH, Wyer PC, Naylor CD, Stiell IG, Richardson WS. Evidence-Based Medicine Working Group. Users' guides to the medical literature: XXII: how to use articles about clinical decision rules. JAMA 2000;284:79-84. doi:10.1001/jama.284.1.79.

18 Douketis J, Tosetto A, Marcucci M, et al. Risk of recurrence after venous thromboembolism in men and women: patient level meta-analysis. BMJ 2011;342:d813. doi:10.1136/bmj.d813.

19 Kyrle PA, Minar E, Bialonczyk C, Hirschl M, Weltermann A, Eichinger S. The risk of recurrent venous thromboembolism in men and women. N Engl I Med 2004;350:2558-63. doi:10.1056/NEJMoa032959.

20 Kearon C, Gent M, Hirsh J, et al. A comparison of three months of anticoagulation with extended anticoagulation for a first episode of idiopathic venous thromboembolism. N Engl J Med 1999;340:901-7. doi:10.1056/NEJM199903253401201.

21 Stein PD, Henry JW, Gottschalk A. The addition of clinical assessment to stratification according to prior cardiopulmonary disease further optimizes the interpretation of ventilation/perfusion lung scans in pulmonary embolism. Chest 1993;104:1472-6. doi:10.1378/ chest.104.5.1472.

22 Wells PS, Anderson DR, Rodger M, et al. Evaluation of D-dimer in the diagnosis of suspected deep-vein thrombosis. N Engl J Med 2003;349:1227-35. doi:10.1056/NEJMoa023153.

23 Schulman S, Kearon C. Subcommittee on Control of Anticoagulation of the Scientific and Standardization Committee of the International Society on Thrombosis and Haemostasis. Definition of major bleeding in clinical investigations of antihemostatic medicinal products in non-surgical patients. / Thromb Haemost 2005:3:692-4 doi:10.1111/j.1538-7836.2005.01204.x

24 Kearon C. Indefinite anticoagulation after a first episode of unprovoked venous thromboembolism: yes. J Thromb Haemost 2007:5:2330-5. doi:10.1111/j.1538-7836.2007.02761.x

25 Konstantinides SV, Torbicki A, Agnelli G, et al. Task Force for the Diagnosis and Management of Acute Pulmonary Embolism of the European Society of Cardiology (ESC). 2014 ESC guidelines on the diagnosis and management of acute pulmonary embolism. Eur Heart J 2014;35:3033-69. doi:10.1093/eurheartj/ehu283.

26 Verhovsek M, Douketis JD, Yi Q, et al. Systematic review: D-dimer to predict recurrent disease after stopping anticoagulant therapy for unprovoked venous thromboembolism. Ann Intern Med 2008;149:481-90, W94.

27 Carrier M, Rodger MA, Wells PS, Righini M, LE Gal G. Residual vein obstruction to predict the risk of recurrent venous thromboembolism in patients with deep vein thrombosis: a systematic review and meta-analysis. J Thromb Haemost 2011;9:1119-25. doi:10.1111/j.1538-7836.2011.04254.x

28 Donadini MP, Ageno W, Antonucci E, et al. Prognostic significance of residual venous obstruction in patients with treated unprovoked deep vein thrombosis: a patient-level meta-analysis. Thromb Haemost 2014:111:172-9. doi:10.1160/TH13-04-0336.

29 Gauthier K, Le Gal G, Shivakumar S, et al. Inter-observer reliability of the HERDO02 clinical decision rule. Thromb Res 2016;141:136-8. doi:10.1016/j.thromres.2016.03.015

30 Le Gal G, Kovacs M), Carrier M, et al. Risk of recurrent venous thromboembolism after a first oestrogen-associated episode. Data from the REVERSE cohort study. Thromb Haemost 2010;104:498-503. doi:10.1160/TH09-10-0685.

31 Le Moigne E, Delluc A, Tromeur C, et all. Risk of recurrent venous thromboembolism among young women after a first event while exposed to combined oral contraception versus not exposed to: A cohort study. Thromb Res 2013:132-51.

Supplementary information: Details of the REVERSE II Study Investigators 